Universidad de Talca - Facultad de Ciencias Jurídicas y Sociales

"Dúctil en el interior; fuerte hacia el exterior. Un análisis de la naturaleza legal de los nuevos instrumentos de derecho laboral internacional"

M. Antonio García-Muñoz Alhambra - Beryl Ter Haar - Attila Kun

\title{
DÚCTIL EN EL INTERIOR; FUERTE HACIA EL EXTERIOR. UN ANÁLISIS DE LA NATURALEZA LEGAL DE LOS NUEVOS INSTRUMENTOS DE DERECHO LABORAL INTERNACIONAL*
}

\author{
M. Antonio García-Muñoz Alhambra* \\ BERYL TER HAAR ${ }^{* * *}$ \\ ATTILA KUN ${ }^{* * * *}$
}

\section{INTRODUCCIÓN}

Las materias y asuntos sociales se regulan mediante instrumentos diversos, entre los cuáles podemos destacar los estándares internacionales, las reglas nacionales, los contratos individuales de trabajo y los convenios colectivos. Muchos instrumentos e instituciones regulan la relación desigual entre el empresario y el trabajador aunque, desde un punto de vista legal, la idea es que la mejor manera de regular estos aspectos es mediante la adopción de normas legalmente vinculantes. Sin embargo, el uso de instrumentos de naturaleza no vinculante desde el punto de vista legal, también llamados instrumentos de soft law, es cada vez más frecuente. ${ }^{1}$ Este desarrollo es considerado generalmente

\footnotetext{
"El presente trabajo ha sido galardonado con el premio "Marco Biagi" 2011, otorgado por la International Association of Labour Law Journals. El original en inglés "Soft on the inside, hard on the outside: an analysis of the legal nature of new forms of International Labour Law", fue publicado en la International Journal of Comparative Labour Law and Industrial Relations, 27, № 4, 2011. Pp. 337-363. La traducción al castellano es de M. Antonio García-Muñoz Alhambra. Colaboración recibida el 10 de abril y aprobada el 27 de abril de 2012.

** Profesor Ayudante de Derecho del Trabajo, Universidad de Castilla - La Mancha, España. Correo electrónico: Manuel.GarciaMunoz@uclm.es.

*** Profesora Ayudante de Derecho del Trabajo, Universidades de Utrecht y Ámsterdam, Holanda. Correo electrónico: berylterhaar@gmail.com.

**** Profesor Asociado. Director del Departamento de Derecho del Trabajo y de la Seguridad Social, Universidad Kàroli Gàspàr de Budapest, Hungría. Correo electrónico: kun.attila@kre.hu.

1 Dupur, R.J, "Declaratory Law and Programmatory Law: From Revolutionary Custom to Soft Law", en AkKerman, R.J.; Krieken, P.J.; PANneborG, Ch.O. (Eds.), Declarations on principles, a quest for world peace (Liber Röling), Sijthof, Leiden, 1977, p. 248; TAYLoR-GobBY, P. "Introduction: Open Markets versus Welfare Citizenship: Conflicting Approaches to Policy Convergence in Europe", en TAylor-Goody, P. (Ed.), Making a European Welfare State?, Blackwell Publishing, Oxford, 2004, p. 1-16; Аввотт, K.W.; SNIDAL, D., "Hard and Soft Law in International Governance", International Or-
} 
indeseable, porque la probabilidad de que el soft law tenga un efecto normativo, en el sentido de diseñar una regulación vinculante, es presumiblemente menorque la de los instrumentos tradicionales o de hard law². Por efecto normativo nosotros entendemos en el presente trabajo dos aspectos: en primer lugar, la generación de un efecto en el orden legal nacional que lleve, por ejemplo, a la adopción o cambio de un acto legal para adecuarlo a los requerimientos del instrumento y, en segundo lugar, la capacidad de asegurar el cumplimiento del contenido normativo del instrumento.

Con independencia de que sea, desde una perspectiva legal, deseable o no, una de las razones para utilizar instrumentos de soft law en la regulación social es el fracaso de los actores públicos, gobiernos y organizaciones internacionales para adoptar reglas legalmente vinculantes en relación con estos asuntos en el nivel transnacional ${ }^{3}$. El mundo de los negocios y también la sociedad civil han reaccionado ante esta disfunción en la "gobernanza" global utilizando instrumentos de autorregulación ${ }^{4}$.

ganization, 54, № 3, 2000, pp. 421-456, especialmente pp. 436-437. Ver en particular, para el Derecho del Trabajo: Morse, D.A. "The Origin and Evolution of the I.L.O. and Its Role in the World Community", Ithaca: New York State School of Industrial and Labor Relations, Cornell University, 1969, pp. 37-73, y Rood, M.G., "Internationalization: A New Incentive for Labour Law and Social Security", en Bellace, J.R.; Rood, M.G. (Eds.), Labour Law at the Crossroads: Changing Employment Relationships. Studies in honour of Benjamin Aaron', Kluwer Law International, La Haya, 1997, pp. 139-154.

2 Klabbers, J. "The Undesirability of Soft Law", Nordic Journal of International Law, 67, 1998, pp. 381 391; Witteveen, W.; VAN Klink, B. "Is Soft Law really Law? A Communicative Approach to Legislation", RegelMaat, 3, 1999, pp. 126-140; BorRÁs, S.; Greve, B. "Concluding remarks: new method or just cheap talk?", Journal of European Public Policy, 11, № 2, 2004, pp. 329-336; DAvIDOv, G. "The enforcement crisis in labour law and the fallacy of voluntarist solutions", IJCLLIR, 26, 2010, pp. 61-82; ComisIÓN EuropeA, Libro Verde sobre la Promoción de un Marco para la Responsabilidad Social Empresarial, COM, 2001. 366 pp.

3 Algunas organizaciones internacionales, como la ICC, la OCDE y la OIT, han adoptado reglas que, sin embargo, no son legalmente vinculantes. Veáse: HorN, N. "International Rules for Multinational Enterprises: The ICC, OECD and ILO Initiatives", American University Law Review, 30, № 81, 1980, pp. 923-940. Para una aproximación más teórica: MACKLEM, P.; TreBilLoCK, M. New Labour Standards Compliance Strategies: Corporate Codes of Conduct and Social Labeling Programs, versión final, Facultad de Derecho, Universidad de Toronto, un trabajo de investigación preparado para la Federal Labour Standards Review, 2006, p. 5, donde citan a Teubner, que introdujo en este contexto el concepto de "derecho global", al que define como un "Nuevo cuerpo legal que emerge de distintos procesos de la globalización en múltiples sectores de la sociedad civil".

${ }^{4}$ STEVIS, D. International framework agreements and global social dialogue: Parameters and prospects, Programa para las Empresas Multinacionales de la OIT, Departamento de Empleo y Desarrollo Empresarial. Documento de Trabajo sobre Empleo № 47, 2010, p. 2, que utiliza el nombre de "regulación cívica"; O'Rouke, D. "Outsourcing regulation: analyzing nongovernmental systems of labour standards and monitoring", The Policy Studies Journal, Vol. 31, № 1. 2003. p. 2, que presenta una perspectiva amplia y lo llama "regulación no gubernamental". Ver también: MuRRAY, J. Corporate social responsibility: an overview of principles and practices, Departamento de Integración Política. Comisión Mundial sobre la Dimensión Social de la Globalización, Oficina Internacional del Trabajo, Ginebra, Documento 
En este contexto, dos instrumentos son de particular interés ya que ambos se enfrentan a la regulación de cuestiones y aspectos sociales en organizaciones multinacionales: los códigos unilaterales de conducta ${ }^{5}$ y los acuerdos marco globales ${ }^{6}$.

Los códigos de conducta son un reflejo del creciente alcance e influencia de las empresas multinacionales ${ }^{7}$. Aunque ampliamente diversos y en continuo cambio", son generalmente definidos como un "conjunto de principios y reglas que gobiernan el modo en que las instituciones sociales deberían actuar frente a terceros interesados y el modo en que estos interesados (especialmente los trabajadores) deberían actuar frente a la institución y entre ellos mismos" ${ }^{\prime \prime}$. Los códigos frecuentemente ponen el énfasis en la responsabilidad social de las empresas multinacionales y son interpretados como un aspecto central y herramienta clave de las agendas de Responsabilidad Social Empresarial (en adelante RSE). Es más, se dice que "el principal objetivo de los códigos corporativos es definir, supervisar y aplicar reglas internas de comportamiento con el objetivo de mejorar la homogeneidad de la organización y la adherencia a obligaciones legales o valores específicos de la empresa y de ese modo reducir los riesgos que puedan surgir de una violación de las leyes nacionales ${ }^{\prime \prime 10}$. Más recientemente, los códigos de conducta se están moviendo desde actos empresariales unilaterales hacia concepciones más complejas y ambiciosas de la RSE ${ }^{11}$.

de Trabajo No 34, 2004, p. 4; Galuin, D. "International Framework Agreements: a reassessment", en Cross-Border Social Dialogue and Agreements: an emerging Industrial relations framework?, Instituto Internacional para los Estudios Laborales, Ginebra, 2008, pp. 15-42.

${ }^{5}$ MACKLEM; TREBILOCK, cit. nota 4; SCHÖMANN, I., y otros, Codes of Conduct and International Framework Agreements: New Forms of Governance at Company Level. Fundación Europea para la Mejora de las Condiciones de Vida y Trabajo, 2008, p. 105.

${ }^{6}$ Gallin, cit. nota 5; Papadakis, K., y otros, Cross-Border Social Dialogue and Agreements: An emerging global industrial relations framework?, ILO, Ginebra, 2008, p. 29, y SteVIS, cit. nota 4.

7 SCHÖMANN; y otros, cit. nota 5.

${ }^{8}$ OIT, Information Note on Corporate Social Responsibility and International Labour Standards, 2003, disponible en internet, en http://www.ilo.org/public/english/standards/relm/gb/docs/gb286/pdf/sdg-4. pdf [visitada el 19.03.2011]; y OCDE, "Codes of Corporate Conduct: Expanded Review of their Contents", Working Papers On International Investment, No 2001/6, p. 33. Veáse también URMINSKY, M. Self-regulation in the workplace: Codes of conduct, social labelling and social responsible investment, OIT, MCC Documento de Trabajo Nº 1, Ginebra, 2002.

${ }^{9}$ CARSOn, A.; SCOtt, M.; McGilL, S. Codes of Conduct in the Private Sector - A Review of the Academic Literature from 1987 to 2007, 2008, p. 2; SeRVAIS, J.M. International Labour Law, Kluwer Law International, La Haya, 2005.

10 Schömann; y otros, cit. nota 5, p. 85.

11 Stevis, cit. nota 4, p. 5. 
Los Acuerdos Marco Globales son considerados como un paso adelante en la evolución de las respuestas de las multinacionales, con origen en ellas mismas, en un esfuerzo por incrementar la legitimidad de sus políticas de RSE $^{12}$. Sin embargo, como rasgo diferenciador, los Acuerdos Marco Globales son el resultado de un diálogo social en el nivel transnacional como un medio para promover y aplicar el núcleo central de los estándares de trabajo de la $\mathrm{OIT}^{13}$. En este sentido, los Acuerdos Marco Globales completan los espacios de disfunción que se producen entre las empresas multinacionales, que son transnacionales, y los interlocutores sociales, tradicionalmente orientados hacia el nivel nacional ${ }^{14}$. Aunque los Acuerdos Marco Globales también presentan una gran variedad, comparten algunas características comunes, basándonos en las cuáles nosotros los definimos como: acuerdos bi o multilaterales entre empresas multinacionales, por una parte, y federaciones sindicales globales, por la otra, en ocasiones acompañadas por sindicatos nacionales y comités de empresa europeos, dirigidos a estimular el diálogo social a nivel mundial y promover el núcleo central de los estándares de trabajo de la OIT ${ }^{15}$.

Aunque estos instrumentos no son adoptados por actores públicos legitimados $y$, consecuentemente, se consideran legalmente no vinculantes en sentido normativo, ambos se dirigen a regular el comportamiento de las empresas multinacionales con respecto a, entre otros temas, las cuestiones laborales. Ser etiquetados como soft law indica que estos instrumentos poseen algunas características legales, tales como normas y estructuras para su cumplimiento, para la regulación de un comportamiento deseado. Entender hasta que punto estos elementos legales están presentes en los instrumentos que nos ocupan (de manera fuerte o débil) y cómo funcionan, mejora no sólo nuestro entendimiento general del funcionamiento legal de los mismos ${ }^{16}$; también mejora nuestra comprensión de las debilidades y fortalezas de los instrumentos en general para generar un efecto normativo ${ }^{17}$. Por lo tanto, supone una aportación para un posterior

\footnotetext{
12 SChÖMAnN; y otros, cit. nota 5, p.3.

${ }^{13}$ Ver, por ejemplo, Galuin, cit. nota 4; SteVIS, cit. nota 4.

${ }^{14}$ CIOSL (Confederación Internacional de Organizaciones Sindicales Libres), disponible en internet, en www.icftu.org/displaydocument.asp? Index=991216332\&Language=EN (visitada el 24.05.2010).

15 Para las diferentes características de las cuales derivamos esta definición: SCHÖMAnN; y otros, cit. nota 5, pp. 21-22; Papadakis, cit. nota 6, pp. 2 y 71; Stevis, cit. nota 4, p. 2.

${ }^{16}$ Aunque son generalmente entendidos como instrumentos de "soft law", existen opiniones doctrinales contrarias. Ver: DAVIDOV, cit. nota 2.

17 Ser considerado soft law no implica necesariamente que no existe alguna influencia normativa. Veáse esto en el contexto del soft law de la Unión Europea: Trubek, D.M; TrubeK, L.G. "The OMC and the Debate over "Hard" and "Soft Law", en Zeituln, J.; Pochet, PH.; Magnussen, L. (Eds.), The Open Method of Co-ordination in Action, P.I.E.-Peter Lang, Bruselas, 2005, pp. 83-103.
} 
fortalecimiento legal de estos instrumentos. En cualquier caso, con la posible excepción de Sobczak y algunas consideraciones generales de tipo descriptivo sobre el soft law ${ }^{18}$, un análisis como este no ha sido realizado.

El objetivo de este trabajo es llevar a cabo un análisis de las fortalezas y puntos débiles desde un punto de vista legal de los Códigos de Conducta y los Acuerdos Marco Globales. Con este objetivo, introducimos un marco que se construye sobre trabajos anteriores de Gamble, Abbott y otros, y Raustialia, y consideraciones de d'Aspremont.

El marco se compone de tres rasgos o dimensiones de legalidad: legalidad en la adopción, contenido o negotium y estructuras para asegurar el cumplimiento. Estas dimensiones son subdivididas a su vez en elementos que devienen determinantes para analizar hasta que punto una dimensión está presente en el instrumento objeto de análisis. La presunción subyacente del marco analítico es que cuando todos los elementos de las tres dimensiones están presentes en su total extensión, el instrumento es entonces hard law; todo elemento que no está presente en su máxima extensión "debilita" el instrumento y lo acerca hacia un concepto de soft law; cuando muchos elementos están ausentes la pregunta que surge es si el instrumento realmente pertenece al ámbito legal. De tal modo que adoptamos una escala legal descendente desde el derecho tradicional o hard law hacia diferentes formas de soft law o incluso de no legalidad en absoluto ${ }^{19}$. Sin embargo, de manera interesante, el análisis muestra dónde se encuentran la debilidad y la fortaleza de un instrumento desde un punto de vista legal, lo que mejora nuestra comprensión de la forma en que regulan el comportamiento de los actores a los que son de aplicación los instrumentos.

\footnotetext{
18 Entre otros muchos: FAwCETT, J.E.S. "The Legal Character of International Agreements", British Journal of International Law, 1953, pp. 381-400; SCHACHTER, O. "The Twilight Existence of Nonbinding International Agreements", The American Journal of International Law, 71, № 2, 1977, pp. 296-304; BAXTER, R. "International Law in her infinite variety", International and Comparative Law Quarterly, 29, 1980, рр. 549-566; Вотне, M. "Legal and Non-Legal Norms - A Meaningful Distinction in International Relations", XI Netherlands Yearbook of International Law, 1980, pp. 65-95; Aust, A. "The Theory and Practice of Informal International Instruments", International and Comparative Law Quarterly, 35, 1986, pp. 787-812; GRUCHALLA-WESIERSKI, T. "A Framework for Understanding Soft Law", McGill Law Journal, 30, 1984/1985, pp. 37-88; LIPSON, C. "Why are some international agreements informal", International Organization, 45, № 4, 1991, pp. 496-498; SHelton, D. Commitment and Compliance. The Role of non-binding Norms in the International Legal System, Oxford University Press, Oxford, 2000.

Algunas consideraciones útiles provienen de aquellos que se oponen a la aceptación de instrumentos no vinculantes legalmente en el ámbito legal. Entre ellos: THürer, D. "Soft Law, eine neue Form von Völkerrecht", Revue de Droit Suisse, 104, № I, 1985, pp. 429-453; KLABBERS, cit. nota 2; D'ASPREMONT, J. "Softness in International Law: A Self-Serving Quest for New Legal Materials", European Journal of International Law, 19, 2008, pp. 1075-1093.

19 En esto seguimos a Abbott, K.W.; Keohane, R.O.; Moravcsik, A.; Slaughter, A.M.; Snidal, D. "The Concept of Legalization", International Organization, 50, № 3, 2000, pp. 401-419.
} 
Tras la introducción del esquema analítico, los Códigos de Conducta y los Acuerdos Marco Globales son analizados a través del mismo. Al ser altamente variables ambos instrumentos, tanto en su contenido como en su estructura, el análisis está basado en definiciones y estudios generales, tal y como será explicado en los respectivos apartados. Dado que el modelo analítico se basa en modelos elaborados para el análisis de instrumentos más tradicionales del derecho internacional, como, por ejemplo, los actos de los actores públicos, mientras que en el presente trabajo nos enfrentamos a la autorregulación de actores no gubernamentales, el análisis adopta en ocasiones una perspectiva amplia y flexible que busca el espíritu de los rasgos y elementos del modelo. Además, hemos de advertir que no se ha realizado para este trabajo un análisis empírico propio de todos los instrumentos, que hubiera sido preferible en relación con algunos elementos del análisis, basándonos por el contrario en las investigaciones ya realizadas (en particular, en relación con los códigos de conducta) y completadas con incursiones en algunos de los instrumentos cuando no existía información disponible (en particular, en el caso de los Acuerdos Marco Globales) ${ }^{20}$. Como ambos instrumentos son utilizados para regular problemas y relaciones laborales y sociales de organizaciones multinacionales, es interesante no sólo analizar su potencial legal individual para generar efecto normativo, sino también comparar las potencialidades y capacidades de ambos instrumentos. Así, las conclusiones alcanzadas no se limitan solamente a resumir los resultados del análisis para la comprensión de los puntos fuertes y las debilidades de estos instrumentos, sino que también posibilitan la comparación de los hallazgos, lo que nos permite un mejor entendimiento de como estos instrumentos regulan conjuntamente los asuntos laborales y sociales en el nivel transnacional.

\section{Modelo analítico para el anÁlisis de nORMAS tRANSNACIONALES}

Aunque es muy complejo crear un modelo definitivo para analizar la calidad legal del derecho internacional, pretendemos introducir un modelo lo más exhaustivo e integral posible, que nos permita un análisis comparativo sistemático de la calidad legal de los instrumentos utilizados para regular cuestiones en el nivel transnacional, que de alguna manera pretende ser un tipo de derecho transnacional. La construcción del modelo se apoya en particular en los marcos elaborados por Gamble ${ }^{21}$, Abbott y otros ${ }^{22}$, y

\footnotetext{
${ }^{20}$ Este es el caso, en particular, del análisis del segundo elemento, contenido o substancia.

21 Gamble, J.K. "The 1982 United Nations Convention on the Law of the Sea as Soft Law", Houston Journal of International Law, 8, 1985, pp. 37-48.

22 Аввот; y otros, cit. nota 19.
} 
Raustiala ${ }^{23}$ y se inspira asimismo en consideraciones llevadas a cabo acerca del "soft law" por d'Aspremont ${ }^{24}$. El marco está compuesto por tres dimensiones de legalidad que son: la legalidad en la adopción de la norma; el contenido o negotium y la estructura que asegura el cumplimiento ${ }^{25}$. Estas dimensiones se subdividen a su vez en distintos elementos que nos determinan hasta qué punto cada dimensión está presente en el instrumento y con qué instensidad. Basándonos en este modelo podemos analizar no sólo la calidad legal de instrumentos legalmente vinculantes, sino también la de aquellos instrumentos que no siendo legalmente vinculantes pretenden alcanzar efecto normativo.

\subsection{Legalidad en la adopción}

Esta primera dimensión se refiere a los aspectos formales de la adopción de un instrumento. Es el elemento más decisivo para responder a la cuestión de cuando un instrumento es o no legalmente vinculante ${ }^{26}$. En esta dimensión podemos distinguir cuatro elementos: la forma del instrumento (instrumentum); el procedimiento de adopción de la norma; la competencia para la adopción de instrumentos legalmente vinculantes y las circunstancias que rodean la creación del instrumento.

La forma del instrumento se refiere al tipo de instrumento que es adoptado. Aunque, tal y como ha sido argumentado por Myers $^{27}$ y Baxter $^{28}$ y confirmado por la jursprudencia del Tribunal de Justicia de la $\mathrm{UE}^{29}$, la forma del instrumento no es conclusiva, no obstante, nos ofrece una primera impresión de lo que los actores han intentado crear.

${ }^{23}$ Raustiala, K. "Form and Substance in International Agreements", American Journal of International Law, 99, 2005, pp. 581-614.

${ }^{24}$ D'ASPREMONT, cit. nota 18.

25 La división de un acuerdo internacional en estas tres dimensiones, aunque para fines diferentes, es realizada por Raustiala, quien perseguía en este intento entender la arquitectura de los acuerdos internacionales, cit. nota 23.

26 Aunque este argumento es utilizado principalmente para negar la existencia del soft law (la ley es "fuerte" o no es ley), la esencia de este argumento es que la ley es legalmente vinculante o no. Veáse: D'ASPREMONT, cit. nota 18; WEIL, P. "Towards Relative Normativity in International Law?", The American Journal of International Law, 77, № 3, 1983, pp. 413-442.

27 Myers, D.P. "The names and scope of Treaties", American Journal of International Law, 51, № 3, 1957, pp. 574-605.

28 BAXTER, cit. nota 18, pp. 549-566.

29 TJUE Case C-311/94, IJssel-Vliet Combinatie B.V. v. Ministerie van Economische zaken [1996] ECR I-5023, par. 44. Otros ejemplos son: TJUE Case C-57/95, France v. Commission [1997] ECR I-1627; TJUE Case C-313/90, CIRFS v. Commission [1993] ECR I-1125; TJUE Case C-366/88, France v. Commission [1990] ECR I-3571. Veáse también KLABbeRS, cit. nota 2, pp. 387-390. 
El segundo elemento tiene que ver con los procedimientos de adopción del instrumento. En general, los instrumentos que se quieren elaborar como legalmente vinculantes tienen que ser adoptados mediante procedimientos específicos que confieren a los mismos la calidad de legales. Estos procedimientos incluyen, por ejemplo, reglas sobre la firma, entrada en vigor, publicación, ratificación, etc. Si un instrumento es adoptado conforme a estos procedimientos, existe mayor probabilidad de que las partes implicadas estén intentando crear un instrumento legalmente vinculante que en los casos en los que no se lleva a cabo la adopción conforme a estos procedimientos.

El tercer elemento es el de la competencia de los actores involucrados en la adopción de un instrumento legalmente vinculante. En esencia, esto supone el hecho de que si un instrumento es adoptado por actores que no son competentes para hacerlo, el instrumento ha sido adoptado al margen de la legalidad y por lo tanto carecerá de vinculatoriedad legal.

El último elemento es el de las circunstancias que rodean la creación del instrumento. Este elemento se refiere a las razones por las cuales los actores que se encuentran en una determinada situación prefieren la adopción de instrumentos que no son legalmente vinculantes frente a elementos que sí lo son. Una de estas circunstancias tiene que ver con la complejidad de la materia a regular. Una materia puede ser compleja porque el contenido de la misma es en sí mismo complejo o porque afecta a muchas otras materias ${ }^{30}$. Otra circunstancia es la confidencialidad tal y como sucede en materias que afectan a la seguridad $^{31}$. Pero también las circunstancias políticas son relevantes para que los actores involucrados elijan o no un instrumentos legalmente vinculante. En particular, cuando parece políticamente imposible alcanzar un acuerdo, es más probable que los actores utilicen como recurso la adopción de instrumentos no vinculantes para intentar superar o evitar un punto muerto ante la posibilidad de un fracaso y la no adopción de ningún tipo de acuerdo o regulación.

\subsection{Substancia / negotium}

La segunda dimensión, substancia o negotium, tiene que ver con la calidad normativa del contenido del instrumento. El contenido son los derechos y obligaciones que el instrumento diseña con el objtetivo de regular el comportamiento de los sujetos que se encuentran dentro de su ámbito de aplicación. Como tal, un instrumento tiene más probabilidades de generar un efecto normativo cuando crea de manera clara y precisa derechos y obligaciones bien definidos que dejan poco margen de interpretación y ambigüedad, cuando las reglas que establece

30 Aввотt; SNIDAL, cit. nota 1.

31 LIPSON, cit. nota 18. 
son coherentes y cuando las mismas se formulan en un lenguaje legal con referencias a un discurso legal ${ }^{32}$. Para su análisis, esta dimensión se subdivide en tres elementos: obligación, precisión y tipo de lenguaje utilizado.

Tal y como muestra Abbott, distintas clases de obligaciones pueden ser establecidas, entre las cuáles (de mayor a menor intesidad obligatoria) encontramos:

- obligaciones incondicionales;

- condiciones implícitas en obligaciones contenidas en un tratado político;

- obligaciones contingentes y cláusulas de escape que permiten reservas nacionales a obligaciones específicas;

- obligaciones exhortatorias, y

- recomendaciones y normas guía ${ }^{33}$.

Qué tipo de obligación ha sido creada puede reconocerse con ayuda de otros dos elementos: precisión y lenguaje utilizado.

El elemento precisión se determina, en primer lugar, por la coherencia interna del instrumento en su conjunto. Esto significa que las normas de los acuerdos no han de ser contradictorias entre sí. En segundo lugar, la precisión se refiere al margen de interpretación y ambigüedad en la defensa de los derechos diseñados o en la ejecución de las obligaciones ${ }^{34}$. Por ejemplo, declaraciones con objetivos amplios e imprecisos o grandes declaraciones de principios se consideran demasiado indefinidas para crear obligaciones incondicionales ${ }^{35}$. Entre otros, Abbott distigue los siguientes cuatro indicadores de precisión (ordenados de mayor precisión a mayor vaguedad):

- reglas determinadas que sólo permiten estrechos márgenes de interpretación;

- reglas que permiten márgenes de interpretación substanciales pero limitados;

- reglas que dejan amplias áreas de discreccionalidad, y

- reglas que contienen "estándares" que son únicamente significativos en referencia a situaciones específicas ${ }^{36}$.

Además, es posible determinar la naturaleza de una obligación analizando el lenguaje que es utilizado para formular derechos y obligaciones. La ley tiene su

\footnotetext{
32 Esta dimensión se apoya con fuerza en la idea subyacente de "concepto de legalidad" tal y como lo explica Аввот, y otros, cit. nota 19.

33 Аввот, y otros, cit. nota 19, p. 410.

${ }^{34}$ En este sentido, cabe precisar que siempre habrá cierto margen de interpretación, no debiendo perderse de vista que cierta ambigüedad y laxitud es inherente a las normas internacionales. FASTENRATH, U. "Relative Normativity in International Law", European Journal of International Law, 1993, pp. 305-340.

35 Parte de la doctrina argumenta que cuando este es el caso, los actores involucrados intentan en ocasiones evitar la vinculatoriedad legal del instrumento. SCHACHTER, cit. nota 18.
}

${ }^{36}$ Аввот, y otros, cit. nota 19. 
propia retórica ${ }^{37}$; además, el tipo de lenguaje que es utilizado es un indicador o señal de la intención de los actores. Por ejemplo, si la intención de los actores es crear un acuerdo legalmente vinculante, probablemente utilizarán un lenguaje con terminología legal que incluya términos como "deberán", "acuerdo", "obligaciones" y "entrada en vigor", en lugar de palabras como "serán", "podrán", "previsiones", "entrar en funcionamiento", que son palabras que denotan una intención de crear algo menos que un acuerdo legalmente vinculante ${ }^{38}$.

\subsection{Estructura}

La tercera dimensión, estructura, se ocupa de "el nivel hasta el cuál los Estados y otros actores delegan autoridad a terceras partes designadas [...] para poner en práctica acuerdos" ${ }^{\prime \prime 39}$. El primer elemento de esta dimensión es la estructura de resolución de conflictos. Se presume que es más fuerte cuando los conflictos se solventan ante los tribunales siendo el extremo más débil las soluciones a través de negociaciones políticas. La fuerza de los organismos judiciales no se basa tanto en sus poderes (constitucionales) de hacer cumplir las normas sino en su función que obliga a necesitar una justificación para alegar incumplimientos, lo que introduce incentivos muy potentes para que los actores adecuen su actuación a la norma ${ }^{40}$. Esto último descansa sobre la asunción de que "palabra y hecho pueden diverger: mientras que la palabra puede adaptarse para coincidir con el hecho, si los procedimientos legales restringen el tipo de argumentos que pueden ser válidamente utilizados, el hecho tiende a adecuarse también"41.

El segundo elemento tiene que ver con el control del cumplimiento. Se considera que existe una estructura fuerte de control del cumplimiento cuando éste queda bajo la tutela de los tribunales de justicia, mientras que se considera que existe una estructura débil cuando hace referencia a métodos como informes de cumplimiento, publicación de información, etc.42.

El tercer elemento de la estructura es la delegación de la posterior elaboración de normativas. Este elemento es útil para el análisis de la naturaleza legal de un instrumento, dado que se presume que sólo un instrumento legalmente vinculante puede delegar la competencia de adoptar en un momento posterior instrumentos vinculantes. Además es un buen indicador del efecto normativo

\footnotetext{
37 Аввот, у otros, cit. nota 19, pp. 409-410.

38 Aust, cit. nota 17, p. 800; Gamble, cit. nota 21, pp. 39-40.

39 Аввот, y otros, cit. nota 19, p. 415; Raustiala, cit. nota 23.

40 Raustiala, cit. nota 23, p. 606.

41 Raustiala, cit. nota 23, p. 606.

42 Raustiala, cit. nota 23, p. 607.
} 
que un instrumento puede generar, ya que la adopción de reglas posteriores, bien sea a través de la jurisprudencia o mediante la adopción de nuevos instrumentos, clarifica y refuerza el comportamiento esperado con la regulación ${ }^{43}$. Solamente la combinación de estos tres elementos nos puede dar una indicación aceptable de la naturaleza legal del instrumento.

\subsection{Modelo analítico}

Basándonos en lo anteriormente expuesto, podemos diseñar un modelo analítico tal y como se muestra en la tabla 1 . El modelo puede ser utilizado tanto para evaluar la naturaleza de instrumentos legalmente vinculantes que son "debilitados" debido a la calidad de sus contenidos o su estructura para asegurar el cumplimiento, así como para el análisis de instrumentos que no son vinculantes legalmente pero se incluyen en el ámbito de lo legal porque se les presume capaces de generar un efecto normativo.

Tabla 1. Modelo de análisis de la naturaleza legal de instrumentos internacionales

\begin{tabular}{|l|l|}
\hline Dimensión & Elementos \\
\hline \multirow{5}{*}{ Legalidad } & $\begin{array}{l}\text { FORMA / INSTRUMENTO } \\
\text { (tratado; declaración; otras formas o instrumentos) } \\
\text { PrOCEDIMIENTO DE ADOPCIÓN } \\
\text { (procedimiento de ratificación nacional; procedimiento } \\
\text { institucionalizado de adopción; otros procedimientos; sin } \\
\text { procedimientos) } \\
\text { COMPETENCIA } \\
\text { (del actor, en particular dentro de contexto de una organización } \\
\text { internacional) } \\
\text { CIRCUNSTANCIAS AMBIENTALES } \\
\text { (COMPlejidad; factores políticos; confidencialidad) }\end{array}$ \\
\hline Substancia / negotium & $\begin{array}{l}\text { TIPO DE OBLIGACIÓN } \\
\text { (incondicional; exhortatoria; política) } \\
\text { PRECISIÓN } \\
\text { (margen de apreciación; coherencia interna) } \\
\text { LENGUAJE UTILIZADO } \\
\text { (palabras Clave; retórica legal o no legal) }\end{array}$ \\
\hline Estructura & $\begin{array}{l}\text { RESOLUCIÓN DE CONFLICTOS } \\
\text { (tribunales, arbitrajes o negociación) } \\
\text { CONTROL DEL CUMPLIMIENTO } \\
\text { (revisión judicial o informes de aplicación) } \\
\text { POSTERIOR REGLAMENTACIÓN } \\
\text { (reglas de aplicación legalmente vinculantes; jurisprudencia; reglas de } \\
\text { interpretación no vinculantes) }\end{array}$ \\
\hline
\end{tabular}

43 Аввот, у otros; cit. nota 19, pp. 416-418. 


\section{Evaluación de la naturaleza legal de los CÓdigos de CONDuCta}

Analizar la naturaleza legal de los códigos de conducta en general es una tarea muy compleja, porque existe muy poca uniformidad entre ellos y porque no deben ser entendidos en términos estáticos, dado que son adaptados y revisados con gran frecuencia. Además, existen enormes diferencias entre los códigos de empresas con sedes en diferentes países y entre los códigos de las empresas en los diferentes sectores económicos. En resumen, los códigos son instrumentos heterogéneos y en constante cambio ${ }^{44}$.

No obstante, en el nivel más general se pueden encontrar algunos elementos comunes. A nivel de regulación es generalmente aceptado que los códigos de conducta son utilizados para completar el vacío de "gobernanza" en el nivel transnacional ${ }^{45}$. En un nivel descriptivo se afirma que "un código de conducta es un nombre dado a un conjunto de principios y reglas que gobiernan el modo en que las instituciones sociales deberían actuar frente a los posibles interesados y afectados y el modo en que los interesados (especialmente trabajadores) deberían actuar frente a la institución y entre ellos mismos" ${ }^{\prime \prime 6}$. Una definición generalmente aceptada la encontramos en Servais, quien define los códigos de conducta en general como "un conjunto de reglas, preceptos o instituciones que pueden ser o no obligatorios desde una perspectiva legal" ${ }^{\prime 47}$. Además, el autor observa que el término es "por costumbre utilizado para referirse a los documentos escritos mediante los cuáles una empresa se compromete a observar cierta política o ciertos principios ${ }^{\prime 48}$. Frecuentemente ponen el énfasis en la responsabildad social de las empresas multinacionales y son interpretados como una herramienta central en las estrategias de RSE. El informe de la Fundación Europea para la Mejora de las Condiciones de Vida y Trabajo, observa que "el principal objetivo de los códigos de conducta es definir, controlar y hacer cumplir reglas internas de comportamiento con el objetivo de mejorar la homogeneidad corporativa y la adherencia a las obligaciones legales o a valores específicos de la empresa, y de esta manera reducir los riesgos de que se pudiesen derivar de una violación de las leyes

\footnotetext{
${ }^{44}$ OIT (1998, Estudio de aproximadamente 258 códigos de conducta y 12 programas de etiquetado social) y OCDE (2001, Estudio de 246 códigos de conducta voluntarios). Los estudios también confirman la enorme diversidad de los códigos de conducta y la falta de consistencia en el tratamiento de los temas en los mismos. Veánse más detalles en: URMINSKY, cit. nota 8.

45 Macklem; Trebilcock, cit. nota 3, pp. 5 y 14; Stevis, cit. nota 4, p. 2; Gallin, cit. nota 4; O'Rouke, cit. nota 4, p. 2; y SCHÖMANN, y otros, cit. nota 5.

${ }^{46}$ Carson, y otros, cit. nota 9, p. 2.

47 Servals, cit. nota 9, p. 99.

48 Servals, cit. nota 9. p. 99.
} 
nacionales" ${ }^{\prime \prime 9}$. Desde el punto de vista del Derecho del Trabajo, los códigos son instrumentos privados, orientados al mercado, que de alguna manera promueven derechos laborales.

El análisis de la naturaleza legal de los códigos de conducta estará basado en estos elementos comunes y nociones generales.

\subsection{Legalidad en la adopción de los códigos de conducta}

Con respecto al primer elemento de legalidad, el instrumento, en teoría, pueden identificarse las siguientes formas de códigos de conducta: acuerdos o convenios colectivos firmados con sindicatos; actos unilaterales empresariales; declaraciones públicas; adicciones a las reglas laborales de la empresa y acuerdos firmados con ONGs ${ }^{50}$. En la práctica, sin embargo, la mayoría de los códigos son adoptados con la forma de instrumentos unilaterales de autorregulación a través de los cuales los principales órganos decisorios de las empresas, establecen reglas de conducta para empleadores y trabajadores (y en ocasiones para proveedores y subcontratistas) que reflejan los principios y valores de la RSE ${ }^{51}$.

No existe un procedimiento uniforme o institucionalizado para la adopción de los códigos de conducta (segundo elemento de legalidad en la adopción). Al contrario, éstos varían significativamenmte de una empresa a otra. Como instrumentos de autorregulación, son establecidos normalmente de manera unilateral por las empresas, o como Wedderburn formulaba a este respecto: "el diálogo social se convierte en un monólogo" ${ }^{\prime 52}$.

Incluso cuando el desarrollo de un código de conducta se lleva a cabo en cooperación con una ONG o un sindicato, o cuando es resultado de algún tipo de diálogo con otros grupos de interesados ${ }^{53}$, no hay un procedimiento formal de adopción que deba seguirse. Esto se deriva del hecho de que las empresas, en general, no tienen obligación legal alguna de negociar con sus empleados o sus representantes antes de adoptar un código de conducta. Aunque debe subrayarse que en algunos países, la legislación laboral nacional requiere que los represen-

\footnotetext{
49 SchÖMAnN, y otros, cit. nota 5, p. 85.

50 Servais, cit. nota 9, pp. 102-103; Urminsky, cit. nota 8, pp. 17-20; Jenkins, R.; Pearson, R.; Seyfang, G. (Eds.), Corporate responsibility and labour rights: codes of conduct in the global economy, Earthscan Publicaciones, London, 2002, p. 28.

51 SCHÖMAnN, y otros, cit. nota 5.

52 MurRay, cit. nota 4.

53 Las negociaciones frecuentemente producen códigos de asociación interempresariales, acuerdos multinivel y acuerdos marco. Ver URMINSKY, cit. nota 8, pp.17-20. Cuando los códigos son el resultado de negociaciones que envuelven a distintos interesados, suelen ser más integrales y exhaustivos e introducen sistemas más estrictos de seguimiento y control que aquéllos que son unilateralmente adoptados por las empresas.
} 
tantes de los trabajadores sean informados y consultados si la dirección de la empresa decide adoptar un código de conducta $^{54}$. Si bien en teoría esa práctica es recomendable ${ }^{55}$, en particular para reforzar la legitimidad de los códigos $^{56}$, en la práctica es bastante excepcional y su impacto es marginal. Es más, se dice que "los representantes de los trabajadores y los sindicatos no juegan en la práctica un papel activo en el proceso de inicio, elaboración y firma de un código de conducta, lo que confirma la naturaleza empresarial de estos textos" ${ }^{\prime 57}$.

Con respecto al tercer elemento de legalidad, la competencia de los actores para adoptar códigos legalmente vinculantes, dos cosas están claras: en primer lugar, las empresas multinacionales no son actores públicos; y, en segundo lugar, no existe ningún marco legal creado por actores públicos que delegue en estas empresas la competencia necesaria para adoptar reglas legalmente vinculantes. Sin embargo, tal y como han dicho algunos autores ${ }^{58}$ " "La regulación no opera únicamente mediante el poder soberano de los estados nacionales sino también a través de entidades diversas como organismos supranacionales, empresas multinacionales y ONGs. Sobre todo, la regulación actualmente depende para su efectividad en gran parte de mecanismos de mercado". Además, Hepple argumenta que "las formas privadas de control social son en ocasiones mucho más importantes para dirigir comportamientos que el cumplimiento de las normas del Estado". También ha sido defendido por algunos que "no hay regulación posible sin auto-regulación" ${ }^{59}$ y como tal, "los códigos de conducta pueden servir como

\footnotetext{
${ }^{54}$ En el año 2000, los tribunales de trabajo franceses decidieron que el comité de empresa debe de ser informado y consultado por la dirección de la empresa si ésta decide adoptar un código de conducta. Veáse SchÖMANN, y otros, cit. nota 5, p. 54.

55 Schwartz, citado por Carson, y otros, cit. nota 9.

${ }^{56}$ Hay algunas nociones alternativas sobre el concepto de legitimidad que pueden utilizarse para defender la legitimidad de los códigos de conducta: "Si aceptamos que la gobernanza sin gobierno supone desafíos para las nociones tradicionales de legitimidad, deberíamos quizás aceptar también un concepto muy amplio de legitimidad, basado en la sociología de las organizaciones, que concibe una norma o institución como legítima si la audiencia relevante la acepta como legítima" (MACKLEM; TREBILCOCK, cit. nota 3, p. 25).

57 SchömanN, y otros, cit. nota 6, p. 3.

${ }^{58}$ Hepple, B. "Does law matter? The future of binding norms", en Polttakis, G. (Ed.), Protecting Labour Rights as Human Rights: Present and Future of International Supervision, Actas del Coloquio Internacional en el 80 Aniversario del Comité de Expertos en la Aplicación de Convenciones y Recomendaciones de la OIT, Ginebra, 24-25 de noviembre de 2006, OIT, 2007; Lobel, O. The Renew Deal: The Fall of Regulation and the Rise of Governance in Contemporary Legal Thought, Universidad de San Diego, School of Law, Legal Studies Research Paper Series, Trabajo de investigación No 07-27, 2005; LanGille, B. "The ILO is not a State, its members are not firms", en PolitakIS, G. (Ed.), Protecting Labour Rights as Human Rights: Present and Future of International Supervision, Actas del Coloquio Internacional en el 80 Aniversario del Comité de Expertos en la Aplicación de Convenciones y Recomendaciones de la OIT, Ginebra, 24-25 de noviembre de 2006, OIT, 2007.

${ }^{59}$ Murray, J. "Corporate Social Responsibility Discussion Paper", Global Social Policy, 4, № 2.
} 
parte de una maquinaria que refuerce la aplicación y legitimidad de las normas legales ${ }^{\prime \prime 6}$. Por otro lado, no hay ninguna duda de que el establecimiento de regulaciones privadas despierta muchas preocupaciones entre los iuslaboralistas que señalan la ambigüa relación entre normas privadas y legislación ${ }^{61}$.

Desde un punto de vista más pragmático, la adopción de un código de conducta puede verse, no como un problema de competencia, sino como un problema de capacidad; se trata de poder, influencia, visibilidad y vulnerabilidad. Así lo sugiere un estudio de la Fundación Europea para la Mejora de las Condiciones de Vida y Trabajo, en el cual se señala que la probabilidad de tener un código de conducta que se ocupe de estándares de trabajo o de las relaciones laborales parece ser mayor en empresas con marcas fuertes, en particular aquéllas en los sectores de productos de consumo y textil ${ }^{62}$. Además, el estudio afirma que el impacto potencial en la imagen corporativa de la violación de derechos sociales fundamentales por las filiales de una multinacional, o incluso por sus proveedores pueden ser incentivos para desarrollar una estrategia proactiva de RSE y, con ella, la adopción de un código de conducta ${ }^{63}$.

Con respecto al cuarto elemento, circunstancias ambientales o contexto, se puede observar lo siguiente. La adopción de un código de conducta es formalmente voluntaria, pero en realidad hay muchos factores complejos por los cuales una empresa se puede ver motivada (o incluso presionada) a elaborar un código. En otras palabras: los códigos de conducta no son sólamente una manifestación de la autorregulación privada de empresas multinacionales, antes al contrario, existe un control difuso de las multinacionales por parte de los mercados y la sociedad civil ${ }^{64}$. Las empresas multinacionales elaboran códigos de conducta por múltiples razones, entre las cuáles podemos encontrar las siguientes:

- elaboración como respuesta a la creciente presión de las ONGs, sindicatos y asociaciones de consumidores;

- para la formalización de las políticas de RSE y su integración en la cultura corporativa;

- para reforzar y mejorar la imagen de marca, la reputación y la cultura corporativa y reducir riesgos de reacciones negativas de los consumidores incrementando al mismo tiempo la legitimidad;

- para comunicar principios y compromisos a los interesados;

${ }^{60}$ Murray, cit. nota 59. p. 7.

61 SChÖMAnN, cit. nota 5, p. 7.

62 Schömann, y otros, cit. nota 5, pp. 35-36. Veáse asimismo: MACKLem; TrebilCock, cit. nota 3, p. 26.

${ }^{63}$ SchÖmann, y otros, cit. nota 5, pp. 35-36.

${ }^{64}$ McBarnet, D.; KurkChiYANM, M. "Corporate social responsibility through contractual control? Global supply chains and other-regulation", en McBarnet, D.; Volculescu, A.; Cambell, T. (Eds.), The new Corporate Accountability - CSR and the law, Cambridge University Press, 2007, p. 91. 
- para guiar el comportamiento y actuación de los trabajadores;

- para reforzar la autodefensa de la empresa ${ }^{65}$ siendo utilizados como escudos frente a presiones políticas;

- para responder a la creciente "crisis de aplicación" en el Derecho Laboral66;

- para responder a las deficiencias de las regulaciones sociales a nivel internacional y al subdesarrollo de la regulación de las actividades de las empresas multinacionales;

- como respuesta a las preocupaciones sobre las deslocalizaciones y restructuraciones y la cada vez mayor necesidad de una regulación social más efectiva de las cadenas de producción globales, etc.

Dado que la globalización está difuminando los papeles respectivos de los Estados y las grandes empresas multinacionales, estas últimas están intentando ganar poder por medio de una autorregulación. En ocasiones, directamente están intentando evitar una regulación estatal. Esta función de vaciamiento (privatización del Derecho) de los códigos podría llegar a socavar las responsabilidades públicas en general ${ }^{67}$. En el caso concreto, las motivaciones pueden variar, diferir de un código a otro y variar en el tiempo.

\subsection{Substancia / negotium de los códigos de conducta}

Así como la tipología de códigos de conducta varía ampliamente, la calidad normativa del contenido de los mismos también lo hace ${ }^{68}$. En general, podemos decir que los códigos de conducta no son obligatorios per se, sin embargo, diversos mecanismos legales pueden relacionarse con los mismos. Es frecuente enfatizar que los códigos voluntarios están de hecho siendo cercados por la regulación legal ${ }^{69}$. McBarnet, por ejemplo, señala que "las empresas sistemáticamente llegan a compromisos en sus códigos de conducta para cumplir con la ley, a pesar de que esto último es, obviamente, una obligación legal"70. De esta manera, las

\footnotetext{
${ }^{65}$ Como subrayan Carson y McGill (cit. nota 9, p. 16), algunos estudios identifican la seguridad legal como uno de los principales propósitos de los códigos de conducta y señalan la existencia de regulaciones incentivadoras en relación con potenciales responsabilidades como factores clave para incluir contenidos específicos en los códigos.

${ }^{66}$ DAVIDOV, cit. nota 2.

${ }^{67}$ Burkett, B.W.; CraIG, J.D.R.; LINK, M., Corporate Social Responsibility and Codes of Conduct: The Privatization of International Labour Law, Canadian Council on International Law Conference, 15 octubre 2004.

68 Schwartz, citado por Carson, y otros, cit. nota 9, p. 10.

${ }^{69}$ McBarnet, D., "Corporate social responsibility beyond law, through law, for law: the new corporate accountability", en McBarnet, D.; Volculescu, A.; Cambell, T. (Eds.), The new Corporate Accountability

- CSR and the law, Cambridge University Press, 2007, p. 31.

70 MCBARnet, cit. nota 69.
} 
empresas tratan de limitar el riesgo de ser sancionadas. En especial, las grandes empresas frecuentemente utilizan los códigos de conducta unilaterales también para imponer sus propias políticas de RSE en los negocios con otros actores, tales como sus propios proveedores y subcontratistas, mediante el uso del control contractual (incorporando los códigos de conducta en los contratos privados) ${ }^{71}$. El resultado es que no solamente existe un espectro amplio de razones para la regulación, sino también oportunidades para modelar la naturaleza y el ámbito de los problemas sociales en los códigos de conducta.

El amplio despliegue de tipos de obligación utilizados en los códigos de conducta puede ilustrarse mediante las fórmulas por las cuales los códigos se aplican (o no) a proveedores ${ }^{72}$ :

- En algunos códigos, la empresa sólo señala compromisos vagos para informar o animar a los proveedores y subcontratistas a que respeten el texto o partes del mismo (sin indicación de las consecuencias de la no adhesión a estos principios);

- Algunos códigos son más estrictos y enfatizan que la adherencia al código es un factor importante para ser elegidos o mantenerse como proveedor o subcontratista;

- Un número limitado de códigos de conducta, particularmente en el sector textil ${ }^{73}$, recogen sanciones precisas (incluyendo la resolución del contrato) para aquéllos proveedores y subcontratistas que no respeten los principios (o algunos principios indicados) del acuerdo.

El contenido de los códigos es distintivo en el sentido de que los mismos son tendenciosos y parciales cuando llegan a la expresión de los derechos que recogen: tienden a centrarse en derechos que se corresponden con situaciones no conflictivas donde tanto la empresa como los trabajadores obtienen beneficios ${ }^{74}$. No en vano, los códigos son en su mayoría elaborados de manera unilateral por la parte empresarial, no por legisladores del ámbito público. Desde un punto de vista objetivo, esta situación provoca dudas sobre la coherencia conceptual y ética de los códigos de conducta. Además, el contendio de los códigos está lejos de ser uniforme porque no sólo se tratan aspectos laborales, sino que también son abordados otros temas típicos de la RSE o de la "ética de los negocios"75.

\footnotetext{
71 MCBARnet, cit. nota 69, p. 42.

72 Para más ejemplos, veáse: SchömAnN, y otros, cit. nota 5, p. 28.

73 Para los ejemplos de los códigos de H\&M, Gap y Levi Strauss \& Co. veáse Schömann, y otros, cit. nota 5, p. 69.

74 SchÖmann, y otros, cit. nota 5, p. 23.

75 Los estudios de la OIT (1998) y la OCDE (2001) así lo muestran. Véase en este sentido URMINSKY, cit. nota 8.
} 
Un examen atento al tipo de derechos reconocidos a los trabajadores muestra que los códigos de conducta hacen referencia a las normas de la OIT, sin embargo, lo hacen de manera poco sistemática. Este es en particular el caso del reconocimiento de la libertad sindical. De acuerdo con ciertos estudios parece existir una clara tendencia hacia referencias más estrictas y completas a los estándares básicos de trabajo en los códigos de conducta ${ }^{76}$, aunque al mismo tiempo muestran que con frecuencia los códigos "redefinen" los derechos humanos, los estándares de trabajo y las definiciones legales mediante la utilización de definiciones propias ${ }^{77}$.

Aunque los códigos varían ampliamente en muchos sentidos en términos de su contenido, en general todos ellos son normativos en el sentido de que "ofrecen pautas sobre cómo se debe actuar"78. En términos de obligaciones "cómo debemos actuar" se expresa generalmente utilizando guías, metas y objetivos. Por consiguiente, su calidad normativa es típicamente soft.

Cuando centramos nuestra atención en el segundo elemento del contenido, precisión, algunos autores señalan que "al localizar la fuente de regulación en la empresa transnacional en sí misma, un código de condutca puede identificar con mucha mayor precisión cómo los estándares generales van a ser aplicados en los centros de trabajo específicos. Como resultado, los códigos pueden resultar adecuados para responder a la complejidad y el cambio continuo de formas flexibles transnacionales de producción mejor que otro tipo de instrumentos que resultan inapropiados para regularlas. Pueden además adaptarse rápidamente a cambios estructurales en una empresa, sector o economía mientras que las normas estatales son más susceptibles de distanciarse de las condiciones estructurales subyacentes" ${ }^{\prime 79}$. Sin embargo, en nuestra opinión, todo esto es válido quizás en un nivel teórico. Dado que no existen indicadores precisos, claros y universales que midan los niveles de lo social, los análisis sobre el grado de cumplimiento y la correcta actuación son, como poco, oscuros y vagos ${ }^{80}$.

Algunos investigadores sugieren que la diferencia entre el espíritu de la ley (ética) y la letra de la ley (legalidad) debería ser considerada cuando se desa-

\footnotetext{
76 SChÖmann, y otros, cit. nota 5, p. 73. Ver también O'ROURKE, cit. nota 4, p. 7.

77 Por ejemplo, códigos de multinacionales tratan la esclavitud infantil en términos variados de precisión, control, edad mínima y sanciones. Veáse Kolk, A.; VAN TulDER, R. "Child Labor and Multinational Conduct: A Comparison of International Business and Stakeholder Codes", Journal of Business Ethics, 36, 2002, pp. 291-301. Veáse también MACKLEM; TreBilcoCK, cit. nota 3, pp. 29-36.

78 Carson, y otros, cit. nota 9, p. 16.

79 Macklem, Trebilcock, cit. nota 3, p. 21.

${ }^{80}$ Ver este tema más elaborado en el punto 4.3.
} 
rrollan los códigos. Para ser útiles, los códigos deberían ser claros, sistemáticos y de aplicación eficaz ${ }^{81}$. En contraste, la práctica revela que la mayoría de los códigos son diseñados utilizando términos abstractos, sin recoger previsiones detalladas sobre cómo las empresas van a aplicarlos y respetarlos.

Sobre la base del tono y el lenguaje utilizado (tercer elemento de la dimensión referente al contenido) en los códigos, es posible hacer una diferenciación entre aquellos que tienen un sentido educativo, inspirador o motivador, y aquellos otros instrumentos que tienen una intención reguladora y prescriben su cumplimiento ${ }^{82}$. Por supuesto, como los códigos son voluntarios, los empresarios formulan el tipo de código que ellos prefieren. Sin embargo, es controvertido qué tipo constituye una herramienta más efectiva para regular los comportamientos.

Parte de la doctrina sostiene que los códigos reguladores que designan conductas particulares como inaceptables son más coherentes con una función disciplinaria, pero requieren definiciones precisas para un buen funcionamiento. Otros, por el contrario, piensan que las funciones educativas y culturales cambiantes de los códigos no quedan bien recogidas por un modelo legalista disciplinario. En ocasiones, la legislación es más útil mientras que otras veces una aproximación basada en valores es más adecuada. Algunos autores señalan que la responsabilidad legal y los requerimientos de la regulación influyen en la existencia y formato de las conductas específicas recogidas en los códigos de conducta ${ }^{83}$.

De manera general podemos identificar las siguientes características sobre el contenido de los códigos de conducta: lenguaje vago, funcional a la empresa, frecuentemente utilizado como estrategias de gestión de recursos humanos y existencia de soluciones retóricas en lugar de substantivas. Algunos autores, entre ellos Sabel, sostienen que existe una cierta tendencia hacia una competición al alza entre los códigos de conducta, especialmente en algunos sectores como la industria del calzado. Este autor señala, no obstante, que tal tendencia es más retórica que basada en contenidos substantivos, en el sentido de que es una tendencia que se observa más en los códigos que en el comportamiento efectivo de las empresas. Pero al mismo tiempo Sabel admite que "las empresas serían extremadamente cínicas o miopes (o ambas cosas) para imponerse estándares cada vez más elevados de conducta a nivel global al mismo tiempo

\footnotetext{
81 Raiborn y PAYNe, citados por CARSON, y otros, cit. nota 9, p. 25.

${ }^{82}$ CARSON, y otros, cit. nota 9, pp. 10 y 15.

83 Para una visión panorámica de la literatura relevante, veáse: CARSON, y otros, cit. nota 9, pp. 15-16.
} 
que presionan consiguiendo condiciones abusivas en los países concretos donde actúan" ${ }^{\prime \prime 4}$.

\subsection{Estructuras para asegurar el cumplimiento de los códigos de conducta}

La principal paradoja de la autorregulación empresarial es el hecho de que "la medida en que las empresas pueden ser consideradas responsables depende directamente de la extensión en la que ellas mismas se consideran vinculadas" ${ }^{185}$. No en vano, como Macklem y Trebilcock subrayan, "las formas diversas de protección de los trabajadores no son algo nuevo para el Derecho del Trabajo". El problema con los códigos de conducta es que la diversidad para la protección está "fundada en la elección empresarial, opuesta a la democrática" ${ }^{86}$. De manera similar a las otras dos dimensiones, la capacidad de las empresas para aplicar y hacer cumplir los códigos de conducta difiere ampliamente. Por ello, el nivel de protección de los trabajadores a través de los códigos de conducta depende del contexto de una empresa determinada. En consecuencia, los códigos de conducta pueden conllevar una "balcanización de las relaciones laborales de acuerdo con su origen en distintas empresas multinacionales" ${ }^{\prime \prime 7}$.

Al llegar a las estructuras para asegurar la aplicación y el cumplimiento de los códigos de conducta descubrimos que generalmente no se preveen mecanismos bien elaborados o cuerpos especializados para la resolución de conflictos (primer elemento de las estructuras para asegurar el cumplimiento). Sin embargo, muchos códigos de conducta diseñan sanciones disciplinarias o incluso civiles para los trabajadores que incumplen sus disposiciones. También muchos códigos incluyen procedimientos de información mediante los cuales los trabajadores son animados a denunciar violaciones del código, frecuentemente mediante la utilización de líneas anónimas ${ }^{88}$. Además, en el contexto de solución de conflictos, Bob Hepple sostiene que la OIT debería facilitar estos contenidos mediante el establecimiento de un organismo de mediación disponible para las multinacionales y los sindicatos para la resolución de posibles

\footnotetext{
${ }^{84}$ SABEL, Ch. "Rolling Rule labor standards: Why their time has come, and why we should be glad of it", en Politakis, G.P. (Ed.), Protecting Labour Rights as Human Rights: Present and Future of International Supervision, Actas del Coloquio Internacional en el 80 Aniversario del Comité de Expertos en la Aplicación de Convenciones y Recomendaciones de la OIT, Ginebra, 24-25 de noviembre de 2006, OIT, 2007, p. 268. Sobre la carrera al alza, veáse también SABel, Ch.; O'ROurke, D.; FunG, A. Ratcheting Labor Standards: Regulating for Continuous Improvement in the Global Workplace, Versión: 2.1, disponible en internet, en http://nature.berkeley.edu/orourke/PDF/RLS21.pdf.

${ }_{85}$ MurRay, cit. nota 4, p. 9.

86 Macklem, Trebilcock, cit. nota 3, p. 27.

87 Boyer, citado en MACKLEM; TReBILCOCK, cit. nota 3, p. 27.

88 SCHÖMANN, y otros, cit. nota 5, p. 4.
} 
controversias sobre la aplicación de códigos de conducta y otros instrumentos (por ejemplo, Acuerdos Marco Globales). Este autor destaca que a largo plazo un Tribunal Internacional del Trabajo, establecido bajo los auspicios de la OIT, será necesario para la resolución de disputas laborales transnacionales ${ }^{89}$.

La aplicación y control de los códigos de conducta (segundo elemento de la estructura para asegurar el cumplimiento) permanece normalmente bajo control empresarial en forma de mecanismos internos de control, frecuentemente delegados a los departamentos de recursos humanos o RSE. Auditores externos, como ONGs, agencias profesionales de auditoría, asociaciones empresariales o sindicatos aparecen ocasionalmente involucrados. Verificaciones y evaluaciones totalmente independientes son poco frecuentes. Una técnica de control utilizada con relativa frecuencia es aquella por la cual los trabajadores tienen el derecho $y$, a veces, incluso, el deber de informar sobre cualquier violación que observen del código, por ejemplo, a través de canales internos o líneas anónimas ${ }^{90}$.

Desde la perspectiva de los actores públicos, la nota positiva es que "el cumplimiento de los estándares de trabajo generado por los códigos de conducta da lugar a una mayor eficacia para el Estado, liberando recusos administrativos escasos que de otra manera tendrían que dedicarse a asegurar el cumplimiento de los requerimientos legales" ${ }^{\prime \prime 1}$.

Pero este proceso de privatización tiene como desventajas la falta de transparencia, rigidez y credibilidad $^{92}$. La investigación confirma estas carencias y muestra que los códigos de conducta unilaterales son de manera generalizada laxos en los procesos de control y presentan un nivel bajo y fragmentario de cumplimiento y de aportaciones substanciales ${ }^{93}$. Además, los códigos de conducta confían en la elección del consumidor (y en sanciones basadas en el mercado) como mecanismo último de asegurar el cumplimiento a gran escala ${ }^{94}$. Para salvar parte de estas críticas, muchas multinacionales han adoptado Acuerdos

\footnotetext{
89 Hepple, cit. nota 58, pp. 230-231.

90 Para más detalles sobre el control, veáse SchÖMAnN, y otros, cit. nota 5, Capítulo 5. Ver también: O'ROUKE, D. "Monitoring the monitors: a critique of corporate third-party labour monitoring", en JENKINS, R.; Pearson, R.; Seyfang, G. (Eds.), Corporate responsibility and labour rights: codes of conduct in the global economy, Earthscan Publicationes, Londres, 2002.

91 MACKLEM; TREBILCOCK, cit. nota 3, p. 21.

92 O'RouKe, cit. nota 90; MACKLEM; TREBILCOCK, cit. nota 3, p. 35. Los incentivos en una empresa para aplicar un código son más débiles que para promulgarlo. De acuerdo con algunas estimaciones, el $80 \%$ de los códigos son en realidad afirmaciones generales sobre la ética de los negocios que no preveen método alguno de aplicación. Esta estimación es de la Organización Internacional de Empleadores.

${ }^{93}$ CAmpBelL, T. "A Human Rights Approach To Developing Voluntary Codes of Conduct for Multinational Corporations", Business Ethics Quarterly, 16, 2006, pp. 225-269.

94 MACKLEM; TREBILCOCK, cit. nota 3, pp. 22 y 42.
} 
Marco Globales precisamente para mejorar la supervisión y control de códigos de conducta adoptados con anterioridad ${ }^{95}$.

Como los códigos de conducta no son legalmente vinculantes como tales, no pueden generar o delegar capacidad normativa posterior (tercer elemento de la estructura para asegurar el cumplimiento). En cualquier caso, se pueden reconocer ciertas formas de capacidad normativa posterior cuando las estrategias de RSE, por ejemplo, los códigos de conducta, inspiran regulaciones posteriores en el sentido de derechos y obligaciones reconocidos en el código que son extendidos a la cadena de producción, esto es, proveedores, subcontratistas, etc. Esta autorregulación no está necesariamente confinada a "inspirar" regulaciones posteriores, sino que puede imponerse a través de mecanismos de control contractual a la cadena de producción. En otras palabras, el cumplimiento con el código de la empresa matriz puede ser articulado en forma de condiciones contractuales o cláusulas en los contratos con subcontratistas y proveedores. De tal manera que, al contrario que las leyes nacionales, los códigos pueden ampliar su ámbito de aplicación sin importar las fronteras cuando son aplicados a las condiciones de trabajo en las cadenas de producción ${ }^{96}$. No sólo las fronteras jurisdiccionales pueden ser superadas con los códigos; igualmente, la separación formal tradicional entre entidades legales diferenciadas puede ser desplazada por la aplicación de los códigos de conducta a las cadenas de producción. Resumiendo, los códigos podrían extender el círculo de personas que están cubiertas por normas laborales ${ }^{97}$.

Parece que esta idea de "cadena de responsabilidades" va más allá de las estrategias de autorregulación de la RSE y está introduciéndose también en el ámbito del pensamiento legal y las políticas públicas. Ejemplos de propuestas de este tipo son las propuestas de la Coalición Europea para la Justicia Corporativa para "Elevar la Responsabilidad Directa de las Empresas Matriz" y para "Establecer un deber de cuidado para la empresa matriz"198, y la Resolución del Parlamento Europeo sobre la responsabilidad social de las empresas subcontratistas en las cadenas de producción ${ }^{99}$. En general, en el contexto del Derecho del Trabajo, esto significa esencialmente que las empresas transnacionales deben

\footnotetext{
95 SCHÖMAnN, y otros, cit. nota 5, p. 3. Sobre las estructuras de aplicación y control de los Acuerdos Marco Globales, véase el punto 4.3.

96 MACKLEM; TrebilCOCK, cit. nota 3, pp. 21 y 26.

97 SoвczaK, A. "Are Codes of Conduct in Global Supply Chains Really Voluntary? From Soft Law Regulation of Labour Relations to Consumer Law", Business Ethics Quarterly, 16, 2006, pp. 167-184.

${ }_{98}$ Coalición Europea por la Justicia Corporativa, Legal Proposals to Improve Corporate Accountability for Environmental and Human Rights Abuses, 2008, disponible en internet, en http://www.corporatejustice.org/IMG/pdf/ECCJ_FairLaw.pdf.

99 Resolución del Parlamento Europeo de 26 de marzo de 2009 (2008/2249(INI)).
} 
asumir una responsabilidad por las condiciones de trabajo en las cadenas de producción y que los contratistas principales deben ser responsables del cumplimiento de las obligaciones laborales de sus subcontratistas ${ }^{100}$.

En el espíritu de la elaboración posterior de regulaciones podemos encontrar que las estrategias de RSE, incluyendo los códigos de conducta, pueden tener algunas repercusiones que pueden generar efectos legales indirectos. El primer posible efecto que puede señalarse en este sentido sucede en la práctica legal. Al ser declaraciones públicas, los códigos de conducta pueden influir no sólo en la opinión pública, sino que abren un espacio para la crítica de la sociedad civil y la imposición de estándares éticos más elevados. Lo que es más, pueden influir a los operadores jurídicos a tener estos estándares autoimpuestos en cuenta en la solución de disputas que afectan a aspectos recogidos en los códigos.

En segundo lugar, el creciente número de códigos de conducta llevan a Bob Hepple a la reflexión de que el Derecho Laboral Internacional debe ocuparse de este fenómeno. Esta regulación no debería estar limitada a un sistema de facilitación de la resolución de conflictos, sino que debería suponer la integración de los estándares de la OIT en los códigos. Una manera en que la OIT podría reforzar esto sería mediante una Convención. Si esta sugerencia se pusiera en práctica podrían etiquetarse como un efecto indirecto en el Derecho Laboral Internacional.

Un tercer posible efecto secundario se puede encontrar en la influencia en la legislación nacional. En este contexto podemos recoger al menos dos nuevos e innovadores desarrollos legales. En primer lugar, están emergiendo iniciativas legales que vienen impulsando directa o indirectamente acuerdos voluntarios y autorregulación. Este es el caso de las regulaciones suplementarias que dejan algún margen a la industria para desarrollar sus propios esquemas regulatorios, tales como códigos de conducta, siempre y cuando recojan ciertas condiciones definidas o guías de contenidos mínimos ${ }^{101}$. Otro ejemplo son los mecanis-

${ }^{100}$ Está claro que con anterioridad a los códigos de conducta las empresas multinacionales argumentaban sistemáticamente que los proveedores no nacionales eran independientes y que la empresa matriz no tenía responsabilidad en absoluto por las conductas de los mismos. Hasta cierto punto la irrupción de los códigos de conducta parece haber modificado esta convicción. Hoy en día existen incluso propuestas legales para elaborar alguna responsabilidad global en este sentido. Cfr. Young, I.M. "Responsibility and Global Labour Justice", The Journal of Political Philosophy, 12, № 4, 2004, pp. 365-388.

101 Ver en este contexto también: Macklem; Trebilcock, cit. nota 3, p. 45, donde sostienen que "el Estado podría elegir dejar algunos elementos de la producción normativa en manos privadas y establecer unas estructuras fuertes de control. O podría dejar la función de control en manos de actores no gubernamentales y crear incentivos para que las empresas maximicen el cumplimiento. Otra opción sería diseñar canales para involucrar a los interesados en la producción normativa, el control y la aplicación para reforzar la legitimidad de las medidas voluntarias". Un ejemplo de esta opción se puede encontrar en Hungría, en el Código Laboral (Norma 22 of 1992), artículo 70/A, que trata de los planes de igualdad de oportunidades. 
mos regulatorios mediante los cuales la adopción de un código de conducta puede ser la base para un sistema selectivo y gradual de sanciones ${ }^{102}$ y puede servir como una herramienta para limitar ciertos riesgos legales, por ejemplo mediante exenciones para los empresarios de ciertos requisitos. Un ejemplo de dicho régimen regulatorio lo encontramos en las Ilamadas Directrices de imposición de penas en los Estados Unidos, que preveen sanciones más leves para las empresas que han adoptado y aplicado códigos de conducta ${ }^{103}$. En este contexto, Davidov menciona, por ejemplo, exenciones en normas de empleo bajo ciertas condiciones para empresas que adoptasen códigos ${ }^{104}$. Ambos tipos de inciativas regulatorias preveen incentivos para los empresarios que ayuden a desarrollar esquemas internos, como los códigos, para asegurar el cumplimiento y la conformidad con la ley. Los Estados pueden reforzar dichas iniciativas utilizando diversos mecanismos, que al final expanden el potencial regulatorio de los códigos de conducta ${ }^{105}$.

En segundo lugar, están emergiendo iniciativas legales para crear lo que las empresas han percibido como "legalmente aplicable de manera voluntaria". En este contexto, algunos tipos de responsabilidad legal se adjuntan a compromisos voluntarios y autorregulatorios, por ejemplo, bajo las normas de protección de los consumidores. Por ejemplo, si una empresa está utilizando su código de conducta como elemento de márketing, pero de hecho no respeta los contenidos del mismo, podríamos estar hablando de publicidad engañosa ${ }^{106}$.

Aunque no queremos defender que estos nuevos desarrollos legales son consecuencia directa del movimiento de los códigos de conducta en general, se pueden constatar desarrollos que indican claramente una convergencia entre enfoques regulatorios y de autorregulación. Como tales, los códigos de conducta de la RSE tienen un efecto indirecto "inspiracional" en los legisladores nacionales, con la consecuencia de que los mismos códigos han llegado a ser progresivamente abordados por la legislaciones nacionales ${ }^{107}$.

\footnotetext{
102 Para el concepto de "regulación receptiva", veáse Hepple, cit. nota 58, pp. 223-224.

103 SchömanN, y otros, cit. nota 5, p. 23. Ver otro ejemplo recogido en este trabajo (p. 35): la Ley Sarbanes-Oxley en los Estados Unidos, adoptada tras el escándalo Enron, impone la adopción de códigos de conducta y procedimientos que hagan posible a los empleados denunciar violaciones de estos códigos por parte de las empresas que aparecen en la lista del mercado de stock americano.

104 DAVIDOV, cit. nota 2.

105 Por ejemplo, mediante leyes de adquisiones socialmente responsables; leyes de información; leyes que requieren a las empresas nacionales con negocios en el extranjero la adherencia a determinados códigos de conducta. Veáse MurRaY, cit. nota 4, sobre los intentos en Australia y los Estados Unidos para diseñar legislación sobre códigos de conducta empresariales.
}

106 Cfr. Artículo 6(2)(b) de la Directiva 2005/29/EC sobre prácticas comerciales desleales.

107 McBarnet, cit. nota 70, p. 31. 
Un cuarto efecto que se puede señalar tiene que ver con las obligaciones de los trabajadores. Los códigos de conducta normalmente contemplan una guía para el comportamiento del trabajador y con frecuencia crean para el mismo nuevos deberes, pudiendo el no cumplimiento de los mismos tener consecuencias en el contrato de trabajo. Muchos códigos de conducta preveen de manera explícita sanciones disciplinarias e incluso civiles para los trabajadores cuyo comportamiento no es conforme a los principios recogidos en el código. En muchos casos, los trabajadores también están obligados a informar de los incumplimientos del código que puedan observar, a través de líneas anónimas ${ }^{108}$.

Junto a los distintos efectos indirectos que señalamos en la esfera legal, tenemos que reconocer las limitaciones obvias ${ }^{109}$ de los códigos de conducta puramente unilaterales, que recientemente han conducido a una intensificación y diversificación de las iniciativas provinientes de la sociedad civil, lo que se conoce en ocasiones como "regulación civil". Al mismo tiempo que la regulación civil está creciendo en importancia, las respuestas empresariales también evolucionan desde códigos de conducta unilaterales hacia enfoques cada vez más complejos y ambiciosos de la RSE ${ }^{110}$.

\section{ANÁlisis de LA NATURALEZA LEGAL DE LOS ACUERDOS MARCO GLOBLALES}

Al igual que los códigos de conducta, los Acuerdos Marco Globales (AMG) presentan múltiples formas y varían considerablemente. Sin embargo, hay algunas características generales sobre las cuáles podemos diseñar una idea general de su naturaleza legal. Simplificando, podemos decir que el propósito y la función principal que desempeñan los AMG es, en general, estimular el diálogo social global entre las empresas multinacionales y los representantes de los trabajadores, así como la promoción de los estándares básicos de trabajo formulados por la OIT"111. Como tales, los AMG formalizan "relaciones dinámicas entre las empresas multinacionales y las federaciones sindicales globales que pueden solucionar problemas laborales en interés de ambas partes ${ }^{\prime 112}$. En segundo lugar, como los códigos de conducta, los AMG completan un vacío de gobernanza en la regulación de los aspectos laborales de las empresas transnacionales. Otra similitud es la ausencia de actores públicos: los AMG

\footnotetext{
108 SCHÖMANN, y otros, cit. nota 5, p. 4.

109 Como se indica en las partes sobre cumplimiento.

110 STeVIS, cit. nota 4, p. 2.

111 SсHÖmANN, y otros, cit. nota 5, pp. 21-22.

112 De acuerdo a la CIOSL (Confederación Internacional de Organizaciones Sindicales Libres), disponible en www.icftu.org/displaydocument.asp? Index=991216332\&Language=EN [visitada el 25-05-2010].
} 
son acuerdos bi o multilaterales entre empresas multinacionales, por un lado, y federaciones sindicales globales, por el otro, frecuentemente acompañadas por Comités de Empresa Europeos ${ }^{113}$. En tercer lugar, el ámbito de los AMG no está necesariamente limitado a los actores directamente envueltos en la adopción de acuerdos, sino que también puede incluir a subcontratistas y proveedores de la mutinacional en cuestión ${ }^{114}$.

Dado que los AMG son diversos y varían, tenemos que hacer algunas precisiones en este sentido. Existen tres enfoques principales en la comprensión de los AMG:

- AMG como el último desarrollo de los instrumentos de RSE hacia enfoques cada vez más complejos y participativos ${ }^{115}$;

- AMG como resultado de políticas públicas en respuesta a la cada vez mayor preocupación de las organizaciones internacionales sobre las externalidades de las actividades económicas de las multinacionales ${ }^{116}$;

- AMG como el resultado de una construcción más amplia de las relaciones laborales transnacionales, que trata de superar el vacío existente de regulación ${ }^{117}$.

Nuestro análisis se centra en este último enfoque de los AMG, como instrumentos que son utilizados para regular las relaciones laborales transnacionales.

\subsection{Legalidad en la adopción de los acuerdos marco globales}

La forma del instrumento (primer elemento de legalidad) para la adopción de los AMG es una cuestión sencilla tal y como se refleja en el nombre: se trata de acuerdos bi o multilaterales. Aunque esto parece simple, todavía existe una discusión acerca de si estos acuerdos son una forma de contrato privado entre las partes involucradas o se trata de convenios colectivos tal y como los cono-

\footnotetext{
113 PAPADAKIS, y otros, cit. nota 6, p. 2.

114 PaPADAKIS, y otros, cit. nota 6, p. 68.

115 STEVIS, cit. nota 4, p. 2.

116 DA COSTA, I.; ReHFELDT, U. "Transnational collective bargaining at company level: historical developments", en Papadakis (Ed.), Cross-Border Social Dialogue and Agreements: an emerging global industrial relations framework?, Ginebra: Oficina Internacional del Trabajo, 2008, pp. 43-64. En este sentido, los autores señalan que "en los años setenta, había una considerable preocupación pública acerca del poder cada vez mayor de las empresas multinacionales, que podía llegar a escapar del control de los Estados nacionales (...) Las empresas multinacionales se enfrentaron a acciones restrictivas por parte de diferentes gobiernos nacionales y movimientos de regulación internacionales. La OCDE trabajó en las Directrices para las Empresas Multinacionales, que fueron adoptadas en 1976, seguidas pronto por la Declaración de Principios Tripartita relativa a las Empresas Multinacionales y la Política Social de la OIT en 1977 (revisadas en 2000 y 2006), y existían negociaciones en marcha para establecer un código de las Naciones Unidas (ONU) para las empreas multinacionales".
}

117 Gallin, cit. nota 4, p. 39. 
cemos en el nivel nacional. En relación con esto último, Sobzack señala que estos acuerdos no deberían ser comparados con los convenios colectivos del nivel nacional, sino que más bien deberían concebirse como un instrumento con sus especificidades propias a nivel internacional ${ }^{118}$.

No existe un procedimiento formal para la adopción de los AMG (segundo elemento de legalidad), sin embargo, siempre aparecen firmados por los representantes de la empresa multinacional y de los representantes de los trabajadores ${ }^{119}$. A pesar de la ausencia de procedimeintos formales, la doctrina ha señalado algunos procedimientos informales de adopción, desde donde se puede distinguir entre reglas relativas a una fase preparatoria y reglas relativas a las fases de negociación y elaboración del texto ${ }^{120}$. Las reglas preparatorias crean las bases por las cuales el completo proceso de negociación y acuerdo de los AMG es llevado a cabo, diseñando, por ejemplo, la posibilidad de reuniones conjuntas, mientras las reglas concernientes a la fase de negociación establecen los parámetros en los cuales tienen lugar las negociaciones, contemplando, por ejemplo, la posibilidad de encuentros por separado o la consulta de expertos ${ }^{121}$. Ambos tipos de procedimientos informales pueden encontrarse en directrices internas o recomendaciones que son acordadas entre las partes ${ }^{122}$.

Debido a la falta de un marco legal en el campo de la negociación colectiva transnacional, ningún poder ha sido explícitamente conferido por ley a ningún actor $^{123}$. Sin embargo, el asunto de la competencia es delicado, dado que los efectos normativos de los AMG dependen de la representatividad de las partes involucradas en las negociaciones ${ }^{124}$. Este es el caso en particular cuando el ámbito de aplicación incluye subcontratistas y proveedores de la multinacio-

\footnotetext{
118 SoвCZAK, A. "Legal Dimensions of International Frameworks Agreements in the field of Corporate Social Responsibility", en PAPADAKIS (Ed.), Cross-Border Social Dialogue and Agreements: an emerging global industrial relations framework?, Ginebra: Oficina Internacional del Trabajo, 2008, pp. 116-121.

119 PAPADAkIs, y otros, cit. nota 6, p. 69.

120 Ver SCHÖMAnN, y otros, cit. nota 5, pp. 49-57.

121 Este es el caso de los "procesos de aprendizaje conjunto entre representantes de los empresarios y trabajadores en el grupo EDF", donde "el primer paso fue un foro de tres días co-organizado por la dirección de la empresa y el Comité de Empresa Europeo en abril de 2003 en el cual las futuras partes firmantes tuvieron la oportunidad de discutir asuntos con ONGs, organizaciones sindicales internacionales y expertos". Veáse ScHÖmanN, y otros, cit. nota 5, p. 53.

122 Ver "Recomendaciones adoptadas por el Comité Ejecutivo en Sevilla, España, mayo de 2007", disponible en formato PDF en ocho idiomas en www.imfmetal.org/index.cfm?c=14863.

123 SOBCZAK, cit. nota 119, p. 117.

124 Ales, E.; Engblom, S.; Jaspers, T.; Laulom, S.; Sciarra, S.; Sobzack, A.; Valdés Dal-RÉ, F. “Transnational Collective Bargaining: past, present and future", Versión final, Comisión Europea, Directorado General de Empleo, Asuntos Sociales e Igualdad de Oportunidades, febrero 2006, p. 21 (disponible en: http:// ec.europa.eu/social/main.jsp?catld=707\&langld=en\&intPageld=214).
} 
nal. En el lado empresarial los problemas de representación surgen cuando el representante no tiene un mandato para negociar en nombre de estos actores. Es ese caso, el AMG no será vinculante para esas partes ${ }^{125}$.

En el lado de los trabajadores surge un problema cuando los trabajadores de las empresas subsidiarias y subcontratistas no están representados por sus propios representantes ${ }^{126}$. Para solucionar estos problemas de representación se intenta involucrar a otras organizaciones en la firma conjunta de los acuerdos, entre otros a los sindicatos nacionales y los Comités de Empresa Europeos, sin embargo, esta solución no es concluyente y presenta además algunas limitaciones $^{127}$.

Los incentivos para la elaboración de un AMG dependen de múltiples factores. Uno de ellos es el vacío de gobernanza o la falta de regulaciones públicas. Otro factor es la distancia entre la actuación mundial de las empresas multinacionales y los marcos de regulación, que permanecen en el nivel nacional, incluyendo la negociación colectiva, de donde surge la necesidad de regular y negociar los aspectos laborales en el nivel transnacional ${ }^{128}$. Junto a estos factores legales podemos encontrar factores "objetivos" que estimulan la adopción de AMG, entre los cuales se encuentran el grado de internacionalización de las empresas, el sector en el cual desarrollan su actividad, la cultura corporativa de la empresa y la calidad del diálogo social en la misma ${ }^{129}$. Otros factores podemos identificarlos en un análisis de los preámbulos de 47 AMG realizado por la Fundación Europea para la Mejora de las Condiciones de Vida y Trabajo $^{130}$. Tres de los factores principales identificados en este análisis fueron: la contribución a la actuación de la empresa y la satisfacción de los accionistas y clientes; la emergencia de un diálogo social internacional que contribuye al desarrollo y la aplicación de derechos sociales fundamentales, en particular aquéllos definidos por la OIT, y la definición o recuperación de estándares legalmente vinculantes y el desarrollo de procedimientos para garantizar su aplicación en las empresas.

\footnotetext{
125 SOBCZAK, cit. nota 119, p. 117.

126 SOBCZAK, cit. nota 119, p. 118.

127 SobCZAK, cit. nota 119, p. 119; y Ales, y otros, cit. nota 125, pp. 21-27, respectivamente.

128 Ver Gallin, cit. nota 4; DA Costa; RehfeldT, cit. nota 116; y SchÖMAnN, y otros, cit. nota 6.

129 STEVIS, cit. nota 4, p. 11, quien sostiene que "los orígenes de los AMG sugieren que las empresas de los países caracterizados por alguna forma de capitalismo negociado tienen más probabilidades de adoptar un AMG que las empresas de países con unas tradiciones más liberales y conflictivas. El hecho de que 57 de 70 AMG hayan sido alcanzados en Europa continental respalda esta hipótesis".

130 SchömanN, y otros, cit. nota 5, p. 39.
} 


\subsection{Substancia o negotium de los Acuerdos Marco Globales}

Los AMG varían enormemente en el tipo de obligaciones que imponen y el lenguaje que utilizan. Incluso dentro de un mismo acuerdo, esto ocurre cuando trata diferentes contenidos que requieren diferentes enfoques. No obstante, en general, se puede constatar que las obligaciones son más bien incondicionales cuando se tratan asuntos relacionados con derechos sociales fundamentales y condiciones de empleo y trabajo, mientras que devienen mucho más laxas e inconcretas cuando se refieren a temas de RSE o ética de los negocios ${ }^{131}$.

Cuando llegamos al tema de la precisión con la que se definen las obligaciones en los AMG encontramos disposiciones muy precisas así como también encontramos ejemplos de otras que son mucho más vagas. Un ejemplo de disposición precisa es la definición de "grupo" en los AMG de PSA:

Este Acuerdo Marco Global se aplica directamente a la totalidad de la división de automoción, investigación y desarrollo, fabricación, ventas y servicios auxiliares así como a las divisiones de finanzas, transportes y logística para las subsidiarias presentes y futuras sobre las cuales la empresa tiene una influencia dominante, bien sea a través del control de la propiedad o, cuando la propiedad

131 Ver, por ejemplo, Ford of Europe agreement upon social rights and social responsibility principles, del 4 de diciembre de 2003, donde podemos encontrar diferencias en el leguaje utilizado cuando se ocupa de representación de los trabajadores: "Ford Europa reconoce y respeta los derechos de sus trabajadores a la libre asociación y a negociar de forma colectiva. La empresa trabajará contructivamente con el Comité de Empresa para promover los intereses de nuestros trabajadores y apoyar la cooperación europea regional de los representantes de los trabajadores. En los lugares donde los trabajadores no estén representados por un cuerpo de representantes de los trabajadores, la empresa ofrecerá oportunidades para recoger las preocupaciones de los empleados. Información y consulta puntuales son un prerrequisito para una comunicación satisfactoria entre la empresa y los representantes de los trabajadores. La información será proporcionada con suficiente antelación para permitir a los representantes prepararse adecuadamente para la consulta. La negociación colectiva sobre las condiciones de trabajo es la expresión de la práctica de la libertad de asociación en el lugar de trabajo y de la responsabilidad de negociar de buena fe para construir relaciones productivas y de confianza en el lugar de trabajo. Incluso cuando aparecen desacuerdos, todas las partes estarán vinculadas por las disposiciones colectivas y legislativas del grupo y se buscará alcanzar soluciones adecuadas". Y cuando se ocupa de la protección medioambiental: "Ford Europa respetará el entorno medioambiental y ayudará a su preservación para generaciones futuras trabajando para conseguir soluciones medioambientales efectivas y factibles y evitando el despilfarro. La empresa trabajará para reducir progresivamente los impactos ambientales de sus actividades en línea con el compromiso adquirido de contribuir al desarrollo sostenible. La empresa analizará, estudiará y gestionará responsablemente el uso de recursos, especialmente el uso de materiales peligrosos y el uso de recursos no renovables. Ford Europa persigue asegurar la coherencia entre sus objetivos sociales, económicos y medioambientales". Acuerdo sobre Derechos Sociales y principios de Responsabilidad Social entre Ford Europa y el Comité de Empresa de Ford Europa. Disponible en internet la base de datos de ETUI-REHS EWC cuya dirección es: http://www.ewcdb.eu/show_company.php?grpco_ID=275 [9 marzo 2011]. 
es menor del $50 \%$, a través de la responsabilidad de la empresa para dirigir los aspectos sociales en las subsidiarias involucradas.

Un ejemplo de una disposición vagamente definida la encontramos en la previsión para la revisión del AMG de Nampak:

Para conseguir los objetivos plasmados en este documento, Nampak y UNI se comprometerán a un diálogo dinámico y se reunirán regularmente, con el propósito de compartir información relevante sobre los negocios y estrategias de Nampak.

También podemos observar grandes diferencias en el lenguaje utilizado entre las diferentes partes de los acuerdos. Sin embargo, en general se puede concluir que, en comparación con otros instrumentos utilizados para regular los asuntos sociales en el nivel transnacional, tales como los códigos de conducta de la RSE, los AMG parecen tener una calidad normativa superior, precisamente porque algunos de ellos incluyen disposiciones que crean obligaciones incondicionales que aparecen formuladas con gran claridad y usan una innegable retórica legal. En los AMG podemos encontrar frecuentemente expresiones como "deber", "acuerdo" u "obligación" con un claro contenido legal. Aunque no podemos ocultar que términos como "será", "debería", "previsiones", etc., que conforman una retórica alejada de lo legal también están presentes, precisamente para evitar la vinculatoriedad legal. En sí mismo esto no es sorprendente; además, el primer tipo de lenguaje suele corresponderse con asuntos de derechos sociales fundamentales y condiciones de empleo y trabajo, mientras que en el segundo caso, nos encontramos ante asuntos de RSE o ética de los negocios.

\subsection{Estructuras para asegurar el cumplimiento de los acuerdos marco globales}

Con respecto a las estructuras para asegurar la aplicación y el cumplimiento de los AMG, podemos señalar que casi todos incluyen mecanismos bien elaborados para este fin y diseñan cuerpos para la solución de conflictos (primer elemento para asegurar el cumplimiento). Muchos AMG prevén el establecimeinto de comités compuestos por representantes de trabajadores y empresarios para la solución de conflictos. Para asegurar un control en este sentido, la mayoría de los AMG establecen que estos comités se reúnan al menos una o dos veces al año ${ }^{132}$. Junto a la posibilidad para los empleados de expresar sus quejas frente a la violación de los derechos creados en el

132 SCHÖMANN, y otros, cit. nota 5, pp. 68-69. 
acuerdo $^{133}$, los AMG incluyen incentivos que estimulan a los trabajadores a informar de violaciones de los acuerdos a sus representantes a nivel local, nacional y transnacional ${ }^{134}$.

Con mayor intesidad que los códigos de conducta, los AMG diseñan elaborados mecanismos de control (segundo elemento para asegurar el cumplimento). Al contrario que en los códigos de conducta, estos procedimientos no están basados en estructuras externas, como auditorías, confiando por el contrario en la negociación y la discusión interna en el marco del diálogo social ${ }^{135}$. De tal manera que se observa que casi todos los AMG establecen procedimientos de control sistemáticos, bien sea a cargo de un comité especial designado por las partes firmantes, que pueden ser utilizados igualmente para la resolución de conflictos ${ }^{136}$, o por los Comités de Empresa Europeos cuando se encuentran involucrados en el concreto $\mathrm{AMG}^{137}$. Algo específico de los AMG con el objetivo de controlar el cumplimiento son las estrategias proactivas dirigidas a crear una cultura de gestión que respete y cumpla con los contenidos de los $\mathrm{AMG}^{138}$ y los disemine entre los empleados de toda la organización ${ }^{139}$.

Una característica atípica para el soft law en general es el hecho de que la mayoría de los AMG incluyen previsiones que diseñan sanciones para asegurar el cumplimiento de los acuerdos por parte de los proveedores y subcontratistas ${ }^{140}$. En este sentido, los AMG van un paso más lejos que los códigos de conducta, ya que no sólo regulan aspectos sociales, sino que también obligan al cumplimento de estas regulaciones a lo largo de toda la cadena de producción. Otra forma de control del cumplimiento característica de los AMG es la posibilidad de renegociar ciertas disposiciones ${ }^{141}$.

\footnotetext{
133 SOBCZAK, cit. nota 119, p. 124.

134 "Normalmente los AMG animan a los trabajadores a contactar con sus representantes locales si descubren alguna violación del acuerdo. Si el problema no puede ser resuelto a este nivel, el trabajador o sus representantes se pueden poner en contacto con el sindicato nacional, que discutirá el problema en la sede nacional de la empresa. Si el problema no puede ser resuelto todavía a ese nivel, las partes firmantes del AMG tratarán de dar solución al conflicto". Veáse: Fundación Europea para la Mejora de las Condiciones de Vida y Trabajo, Codes of Conduct and international framework agreements: New forms of governance at company level, 2008, p. 70.

135 SCHÖMANN, y otros, cit. nota 5, p. 63.

136 SOBCZAK, cit. nota 119, p. 125. A veces la supervisión es confiada a los Comités de Empresa. Ver SCHÖMANN; y otros, cit. nota 5, p. 63.

137 SCHÖmANN, y otros, cit. nota 5, p. 63.

138 SCHÖmAnN, y otros, cit. nota 5, p. 70.

139 SOBCZAK, cit. nota 119, p. 124.

140 SCHÖmAnN, y otros, cit. nota 5, p. 69.

141 SchÖmanN, y otros, cit. nota 5, p. 63. Ver también el segundo ejemplo en el punto 4.2.
} 
Aunque los AMG son generalmente concebidos como legalmente no vinculantes, pueden sin embargo generar regulaciones posteriores (tercer elemento de la estructura). Los grandes principios, tales como los estándares laborales de la OIT, recogidos en los AMG, son posteriormente desarrollados en procesos negociadores en los niveles local o nacional ${ }^{142}$. En este sentido, los AMG pueden ser transpuestos en el orden nacional dándoles el mismo estatuto legal que a los convenios colectivos nacionales cuando un sindicato nacional firma el $\mathrm{AMG}^{143}$.

Al igual que los códigos de conducta, debemos señalar que los AMG pueden generar múltiples influencias que indirectamente den lugar a efectos en lo legal. El primero de estos efectos es que los AMG tienen un impacto en los estándares de trabajo de la OIT, dado que reafirman y en ocasiones dan a conocer estos derechos y obligaciones ${ }^{144}$. En segundo lugar, tal y como es destacado por algunos autores, los AMG pueden tener un impacto en las relaciones laborales y el diálogo social en los niveles local y nacional ${ }^{145}$. Además, los AMG pueden tener todos los efectos que ya hemos visto para los códigos de conducta. Por lo tanto, los AMG pueden influenciar la cultura empresarial bien a través de cambios formales algunos de los objetivos y principios empresariales o reforzando enfoques que aporten otros puntos de vista ${ }^{146}$.

\section{CONCLUSIONES}

Con la internacionalización de las empresas, temas regulados tradicionalmente por el Derecho del Trabajo, como las condiciones de trabajo, han superado las fronteras nacionales y se han convertido en objeto del ámbito legal transnacional. Sin embargo, debido al fracaso de los actores públicos para adoptar reglas legalmente vinculantes que gobiernen estos asuntos laborales, actores privados, tales como las empresas multinacionales y las federaciones sindicales globales han tomado la inciativa e introducido nuevas formas de regular los asuntos laborales en el nivel transnacional. Dos de estas formas, los códigos de conducta como parte de las estrategias de RSE de las multinacionales, y los AMG alcanzados entre las multinacionales y las federaciones sindicales globales, frecuentemente acompañadas por los Comités de Empresa Europeos, han sido analizados en este trabajo. Este análisis ha tenido como objetivo en-

142 SOBCZAK, cit. nota 119, p.121.

143 SOBCZAK, cit. nota 119.

144 SChÖMANN, y otros, cit. nota 5, p. 73.

145 SchÖmANN, y otros, cit. nota 5, p. 77.

146 SCHÖmANN, y otros, cit. nota 5. 
tender los puntos fuertes y debilidades de la estructura de estos instrumentos desde un punto de vista legal. Para su realización hemos introducido un modelo formado por tres dimensiones de legalidad y posteriormente dividido en varios elementos que representan una graduación legal que se mueve desde el derecho fuerte o tradicional hasta diferentes formas de soft law o que quedan fuera de la legalidad.

El modelo analítico presenta dos limitaciones. En primer lugar, está basado en concepciones "tradicionales" de la ley, por ejemplo, normas adoptadas por los actores públicos, mientras que el análisis se aplica a formas de autorregulación Ilevadas a cabo por actores no gubernamentales. Para solventar esta limitación, el análisis no interpreta de manera estricta los elementos del modelo, sino que se practica un enfoque más flexible. Otra limitación de nuestro análisis es la falta de una investigación empírica requerida para un análisis más exhaustivo y sistemático. Nosotros hemos optado por apoyarnos en los trabajos de otros autores, en particular en el análisis de los códigos de conducta completados con incursiones en los instrumentos cuando no había literatura disponible.

Con respecto a las tres dimensiones de legalidad propuestas, creemos que desde una perspectiva estricta de derecho público, ambos instrumentos son adoptados fuera del marco legal, porque sólo los actores públicos tienen capacidad para adoptar legitimamente normas que sean legalmente vinculantes para terceras partes, como es el caso de los códigos de conducta y los AMG frente a trabajadores, subcontratistas y proveedores. Sin embargo, cuando se aplica un enfoque más abierto, podemos encontrar argumentos para considerar que los actores involucrados tienen al menos competencia material para adoptar estos instrumentos. En primer lugar, existe un vacío de regulación. En el caso de los códigos de conducta y los AMG, este espacio es ocupado por las multinacionales y las federaciones sindicales globales. Visto desde un principio de subsidiariedad, las multinacionales y las federaciones son los actores más cercanos a los problemas laborales transnacionales y, por lo tanto, podría argumentarse que son competentes para (auto)rregular estos aspectos. En segundo lugar, en el nivel nacional es generalmente aceptado que ambos lados de la industria concluyan acuerdos sobre temas laborales, estando los actores incluso organizados a nivel regional, como en la Unión Europea, bajo los procesos de diálogo social o en el nivel internacional con la OIT. Cuando este principio es Ilevado un paso adelante parece casi obvio que ambos lados de la industria se encuentren en el nivel transnacional para concluir acuerdos sobre aspectos laborales. Con respecto a los AMG, podemos destacar que, en particular, las federaciones sindicales globales tratan de reforzar su competencia involucrando a los Comités de empresa europeos y los sindicatos nacionales en su firma.

Con respecto a la calidad normativa del contenido o substancia de estos instrumentos tenemos que hacer una distinción entre los mismos. En general, 
los códigos de conducta tienden a incluir estándares de trabajo autodefinidos que se corresponden con situaciones no conflictivas. Por lo tanto, no sorprende que los mismos hayan sido recogidos en directrices que expresan como las empresas deberían actuar y no como van a actuar. Es más, normalmente se caracterizan por el uso de un lenguaje vago, funcional a la empresa, y utilizado frecuentemente como herramienta de marketing que ofrece soluciones retóricas frente a contenidos substantivos. En consecuencia, los códigos de conducta apenas son algo más que declaraciones de intenciones, lo que, en los términos de nuestro análisis, debilita considerablemente su calidad normativa. Los AMG nos ofrecen una impresión difusa en cuanto a su contenido, que varía desde vagas recomendaciones y directrices que evitan la retórica legal hasta derechos incondicionales enunciados de manera precisa y obligaciones que no tratan de escapar de la apariencia legal. Este último tipo de disposiciones son utilizadas especialmente para la formulación de estándares y condiciones de trabajo. Nuestra conclusión es, por lo tanto, que el contenido de los AMG es sustancialmente más fuerte que el de los códigos de conducta.

Aunque la paradoja de la autorregulación es que los actores involucrados sólo pueden ser responsables hasta el punto en que ellos mismos quieren vincularse, la parte más interesante de ambos instrumentos son las estructuras que han creado para asegurar el cumplimiento de los mismos. El análisis muestra que ambos instrumentos han organizado algún tipo de "responsabilidad en cadena". Por ejemplo, utilizando el control contractual privado, lo que significa que el cumplimiento con el instrumento de la empresa matriz puede ser impuesto y articulado como un requerimiento o claúsula contractual en los contratos con los subcontratistas y proveedores. El análisis muestra también que no es infrecuente en este tipo de control utilizar sanciones tan graves como la terminación del contrato entre la empresa matriz y el subcontratista o proveedor. Esto es destacable porque una de las principales características del soft law en general es que la obligación de cumplimiento nunca está reforzada por revisión judicial o sanciones. Desde un punto de vista pragmático, la sanción consistente en la terminación del contrato puede ser un mecanismo más fuerte en la práctica que la revisión judicial, dado que supone un fortísimo incentivo económico para el cumplimiento de las normas.

Además, cuando adoptamos otra vez un enfoque amplio y abierto desde el modelo analítico, descubrimos la importante capacidad de ambos instrumentos para generar efecto normativo, una capacidad que hemos explicado a través de los efectos que generan en el ordenamiento. Hemos visto distintas maneras en las que pueden generarse estos efectos. Éstas varían desde los tradicionales efectos del soft law, como el papel interpretativo en la revisión judicial y la apertura de caminos para la adopción de normas tradicionales en los niveles internacional y nacional, hasta desarrollos más innovadores como regulación 
suplementaria sobre autorregulación que abra el camino a la industria para desarrollar sus propios regímenes reguladores y la incorporación de obligaciones para los trabajadores en los contratos de trabajo.

Cuando tomamos lo anterior en consideración, nuestra conclusión es que, si nos basamos en un análisis estricto mediante la aplicación del modelo analítico propuesto, ambos instrumentos, los códigos de conducta y los AMG son muy débiles: son elaborados fuera del marco legal dado que los actores involucrados carecen de competencia formal; la calidad normativa de los mismos varía desde muy vaga hasta precisa; y la estructura para asegurar el cumplimiento aparece limitada, sobre todo en los códigos de conducta a estructuras internas de solución de conflictos y control de la actuación general. Sin embargo, cuando adoptamos un enfoque más amplio y abierto en nuestro análisis, ambos instrumentos parecen ser mucho más fuertes hacia el exterior de lo que su estructura formal sugiere: los actores involucrados tienen una competencia inherente para tratar de los asuntos laborales; aunque el contenido normativo es vago, nos da las pautas de cómo los actores a los que se les aplica deberían actuar; como resultado de las expectativas en los comportamientos creadas por los contenidos, estos instrumentos son capaces de generar efectos que pueden tener consecuencias importantes, como la resolución contractual.

Por lo tanto, nuestra conclusión fundamental es que, aunque la estructura formal de estos instrumentos es débil o dúctil, su efecto normativo hacia el exterior puede ser fuerte. 
